\title{
REDUCTION OF OPEN MAPPINGS
}

\author{
R. F. WILLIAMS
}

In $1936 \mathrm{G}$. T. Whyburn [8] defined an irreducible mapping to be a mapping $f$, of a compact metric continuum $A$ onto $B$, such that no proper sub-continuum of $A$ maps onto $B$ under $f$. Similarly, assuming $A$ to be only compact and metric, he defined $f$ to be strongly irreducible provided that no closed proper subset of $A$ maps onto $B$. J. Rozanska [7] independently defined this second type of mapping in 1937. However, neither of these early papers studied these concepts per se, except that Whyburn pointed out that the Brouwer Reduction Theorem does guarantee that either of these two phenomena can be obtained by perhaps reducing $A$ to a subset, $A^{\prime}$.

In 1939 Whyburn published a paper [9] specifically about these mappings and proved that for $f$ to be strongly irreducible it is necessary and sufficient that the set $D$, of all points at which $f$ is one-toone, be dense in $X$. Also proved were certain other characterizations and another theorem which is of special interest here. This theorem fits into a sequence of theorems beginning with a question raised by W. A. Wilson [11] in 1935.

As a setting for these theorems, suppose that $f$ is an open mapping of a compact metric space $X$ onto $Y$. Wilson's question can be asked as follows: if $Y$ is an arc, can $f$ be monotone and irreducible though not a homeomorphism? B. Knaster [5], in 1935, published an example where this is indeed the case. However, in this example the inverses of points of $Y$ are mixed, some being arcs and others indecomposable continua. Knaster then posed the question as to whether there exists an example in which all inverses are homeomorphic, and in particular, are all arcs.

The question concerning arcs as inverse sets was answered in the negative by $\mathrm{E}$. E. Moise [6] in 1949 and the more general question is answered in the affirmative (as Eldon Dyer has pointed out) by an example that R. D. Anderson [1] has announced in another connection, in which the inverses of points are pseudo-arcs. In the meantime certain gaps between these two results have been filled in by authors who were concerned with slightly different hypotheses on $X, Y$, and $f$. In 1939 Whyburn [9] proved that if $Y$ is unrestricted, $f$ is light,

Presented to the Society, December 29, 1953; received by the editors October 25, 1954 and, in revised form, May 20, 1955. 
and $X$ is a semi-locally-connected continuum, then $f$ has to be a homeomorphism to be irreducible. M. E. Hamstrom [4], in 1953, proved that in case $Y$ is an arc, the negative answer of Moise still holds if the condition on the inverse of points is relaxed to allow arbitrary locally connected continua. Then Dyer [3] and the author independently proved that the answer is still in the negative for arbitrary $Y$, if the inverse of points are decomposable continua.

This paper includes a slightly stronger form of the theorem mentioned above in connection with Dyer and an analogous result for light open mappings, though as is also shown, some restriction has to be made on $Y$. The last theorem states that if $Y$ is perfect and the inverse of each point of $Y$ is a locally connected continuum, then there exists a closed subset, $X^{\prime}$, of $X$, such that $f \mid X^{\prime}$ is (1) monotone and (2) strongly irreducible onto $Y$. In conclusion it is perhaps interesting to note that though property (1) is inducible, the irreducibility obtained is relative to property (2), and thus is not a direct consequence of the Brouwer Reduction Theorem.

Though the concept of irreducible mappings is implicit in all that follows it seems inconvenient to use this terminology explicitly.

Standing Hypothesis. Throughout this paper $X$ and $Y$ are compact metric spaces and $f$ is an open mapping of $X$ onto $Y$. Under this hypothesis $X$ is perfectly separable and $\left\{N_{i}\right\}_{i=1}^{\infty}$ will denote a countable basis of neighborhoods in $X$ such that the diameter of $N_{i} \rightarrow 0$ as $i \rightarrow \infty$. The distance function $\rho$ will be used for both $X$ and $Y$.

THEOREM 1. If in addition to the standing hypothesis, $R$ is a nonempty open subset of $Y$ such that for each $y \in R, f^{-1}(y)$ is a decomposable continuum, then there exists a closed proper subset, $X^{\prime}$, of $X$, such that $f \mid X^{\prime}$ is monotone onto $Y$ and such that $X^{\prime} \supset X-f^{-1}(R)$.

Proof. Let $Q=\left\{(h, i, j, k) \mid \bar{N}_{h} \subset N_{j}, \bar{N}_{i} \subset N_{k}\right.$, and $\left.\bar{N}_{j} \cdot \bar{N}_{k}=0\right\}$. For each $q=(h, i, j, k) \in Q$, let $Y_{q}=\left\{y \mid y \in R, f^{-1}(y)=L+M\right.$, where $M$ is a continuum, $\bar{N}_{h} \cdot M \neq 0, \bar{N}_{i} \cdot L \neq 0$, and $\left.N_{j} \cdot L+N_{k} \cdot M=0\right\}$. Then $\bigcup_{q \in Q} Y_{q}=R$, because for each $y \in R, f^{-1}(y)$ is decomposable. Furthermore, for $q=(h, i, j, k) \in Q, Y_{q}$ is closed relative to $R$. For suppose that $\left\{y_{\alpha}\right\}_{\alpha=1}^{\infty}$ is a sequence of points of $Y_{q}$ converging to $y \in R$. Then for each positive integer $\alpha, f^{-1}\left(y_{\alpha}\right)=L_{\alpha}+M_{\alpha}$, where $M_{\alpha}$ is a continuum, and (1) $\bar{N}_{h} \cdot M_{\alpha} \neq 0$, (2) $\bar{N}_{i} \cdot L_{\alpha} \neq 0$, and (3) $N_{j} \cdot L_{\alpha}+N_{k} \cdot M_{\alpha}=0$. For some subsequence, $\left\{\alpha_{p}\right\}_{p=1}^{\infty}$, of the index $\alpha$, both $\left\{L_{\alpha_{p}}\right\}_{p=1}^{\infty}$ and $\left\{M_{\alpha_{p}}\right\}_{p=1}$ converge to limits, say $L$ and $M$ respectively. Then, as $f$ is open, $f^{-1}(y)=L+M$. Furthermore $M$ is a continuum and conditions (1), (2), and (3) are satisfied by $L$ and $M$, as they are satisfied by $L_{\alpha}, M_{\alpha}$ for each positive integer $\alpha$. Therefore $y \in Y_{q}$. 
Therefore as there are only countably many elements in $Q$, some $Y_{q_{0}}$ contains an open subset, $R^{\prime}$, of $R$. For each $y \in R^{\prime}$, let $f^{-1}(y)$ $=L_{y}+M_{y}$, where $M_{y}$ is a continuum and $L_{y}$ and $M_{y}$ satisfy conditions (1), (2), and (3), for $q=q_{0}$. Let $(H, I, J, K)$ denote the terms of $q_{0}$, let $X^{\prime \prime}=X-f^{-1}\left(R^{\prime}\right)+\cup_{y \in R^{\prime}} M_{y}$, and let $X^{\prime}=\bar{X}^{\prime \prime}$. Then $f\left(X^{\prime}\right)=Y$, $X^{\prime} \subset X-N_{K}$, and $X^{\prime} \supset X-f^{-1}(R)$.

Furthermore, $f \mid X^{\prime}$ is monotone, for suppose $y \in R^{\prime}$ and $x \in f^{-1}(y) \cdot X^{\prime}$. Then, either $x \in M_{y}$, or for some sequence $\left\{x_{\alpha}\right\}_{\alpha=1}^{\infty}$, of points of $R^{\prime}$, $x \in \lim _{\alpha \rightarrow \infty} M_{y_{\alpha}}$. But $\lim _{\alpha \rightarrow \infty} M_{y_{\alpha}} \supset f^{-1}(y) \cdot \bar{N}_{H}$ which is nonempty. Therefore each point $x \in f^{-1}(y) \cdot X^{\prime}$ lies in a component of $f^{-1}(y) \cdot X^{\prime}$ which contains the nonempty set, $M_{y} \cdot \bar{N}_{H}$. Thus $f \mid X^{\prime}$ is monotone.

The next result, presented here as a corollary to Theorem 1, was obtained independently by Dyer [3] and the author. Dyer's proof is of a different nature and somewhat longer than these arguments.

CoROllary (Dyer [3]). If, in addition to the standing hypothesis, $Y$ is a continuum and for each $y \in Y, f^{-1}(y)$ is a decomposable continuum, then $X$ is not irreducible.

Proof. Suppose $A, B \in X$. Let $R=Y-\{f(A), f(B)\}$. By Theorem 1 there exists a proper closed subset $X^{\prime}$, of $X$, containing $X-f^{-1}(R)$, such that $f \mid X^{\prime}$ is monotone onto $Y$. But as the continuum $Y$ is the monotone continuous image of $X^{\prime}$, then $X^{\prime}$ is a continuum. As $X^{\prime} \supset X-f^{-1}(R), A, B \in X^{\prime}$.

TheOREM 2. If, in addition to the standing hypothesis, $f$ is light, $Y$ is connected, $Y$ is semi-locally-connected at a point $y_{0} \in Y$, and $f^{-1}\left(y_{0}\right)$ is nondegenerate, then there exists a proper sub-continuum $X^{\prime}$ of $X$ such that $f\left(X^{\prime}\right)=Y$.

Proof. There exists a neighborhood $N$ of $y_{0}$ such that $\Gamma$, the collection of all components of $Y-N$, is finite, and such that $f^{-1}(N)$ $=U+V$, where $\bar{U} \cdot \bar{V}=0$, and $f(U)=f(V)=N$. Then if $\gamma \in \Gamma$, each component of $f^{-1}(\gamma)$ maps onto $\gamma$ under $f$. Let $\gamma_{1}$ denote an element of $\Gamma$; there exists a component $X_{1}$ of $f^{-1}\left(\gamma_{1}\right)$ which intersects $\bar{U}$. Let $X^{\prime}$ denote the component of $X-V$ which contains $X_{1}$.

Then $f\left(X^{\prime}\right)$ contains $Y-N$, for suppose the contrary. Then if $\gamma \in \Gamma$, either $\gamma \subset f\left(X_{1}\right)$ or $\gamma \cdot f\left(X_{1}\right)=0$. Let $Y^{\prime}=(Y-N)-f\left(X^{\prime}\right)$. As $Y^{\prime}$ is the union of the elements of a sub-collection of $\Gamma, Y^{\prime}$ is closed. Hence there is a continuum lying in $\bar{N}$ which intersects $f\left(X^{\prime}\right)$ and $Y^{\prime}$. Since $f \mid \bar{U}$ is open onto $\bar{U}, \bar{U}$ is not the sum of two mutually exclusive sets, one containing $X^{\prime} \cdot \bar{U}$ and the other containing $f^{-1}\left(Y^{\prime}\right) \cdot \bar{U}$. Therefore, there exists a continuum $M^{\prime} \subset \bar{U}$ which intersects $X^{\prime}$ and $f^{-1}\left(Y^{\prime}\right)$, contradicting the definition of $Y^{\prime}$. 
If $y^{\prime} \in \bar{N}$, there is a continuum $M^{\prime} \subset \bar{N}$ containing $y^{\prime}$ and a point of $Y-N \subset f\left(X^{\prime}\right)$. As before, there exists a continuum $M \subset \bar{N}$ which intersects $X^{\prime}$ and $f^{-1}\left(y^{\prime}\right)$. Hence $y^{\prime} \in f\left(X^{\prime}\right)$.

EXAMPLE. There exists a nonhomeomorphic (3-to-1), light open mapping $f$ of a compact continuum $\Sigma_{2}$ onto itself such that no proper subcontinuum of $\Sigma_{2}$ maps onto $\Sigma_{2}$ under $f$.

Proof. The continuum $\Sigma_{2}$ has been studied by several authors [2] and is sometimes called a "two-solenoid." It can be described as follows: Let $C$ denote the complex unit circle. Then $\Sigma_{2}=\left\{\left(c_{0}, c_{1}\right.\right.$, $\left.c_{2}, \cdots\right) \mid$ for each integer $i \geqq 0, c_{i} \in C$ and $\left.c_{i+1}^{2}=c_{i}\right\}$, where a subset $N$ of $\Sigma_{2}$ is open if and only if there exists a neighborhood $N^{\prime} \subset C$, and a positive integer $i$ such that $N=\left\{c \mid c \in \Sigma_{2}\right.$ and $\left.c_{i} \in N^{\prime}\right\} . \Sigma_{2}$ can be embedded in $\mathbf{E}_{3}$ and is a compact metric indecomposable continuum, each nondegenerate proper sub-continuum of which is an arc.

For each $a=\left(a_{0}, a_{1}, a_{2}, \cdots\right) \in \Sigma_{2}$ let $f(a)=\left(a_{0}^{3}, a_{1}^{3}, a_{2}^{3}, \cdots\right)$. Then $f$ is continuous and $f\left(\Sigma_{2}\right) \subset \Sigma_{2}$. From the fact that $\Sigma_{2}$ is compact it follows that if $b \in \Sigma_{2}, z \in C$, and $z^{3}=b_{n}$, then there exists a point $a \in \Sigma_{2}$ such that $a_{n}=z$ and $f(a)=b$. This last implies that $f$ is 3-to-1 onto $\Sigma_{2}$ and, in conjunction with the fact that power maps are open, it implies that $f$ is open. Lastly, if $M$ is a proper sub-continuum of $\Sigma_{2}, M$ is either a point or an arc. Hence $f(M)$ is locally connected and therefore $f \mid M$ is not onto $\Sigma_{2}$.

In [10], the following definition is given, in which the standing hypothesis and notation are used: an open subset $V$ of $X$ will be said to have property $\mathrm{P}$ if and only if (1) $f \mid \bar{V}$ is monotone, and (2) if $y \in f(\bar{V})$, then there exists a point $x \in f^{-1}(y) \cdot \bar{V}$ and a neighborhood $N$ of $x$ such that $f^{-1} f(V) \cdot N \subset V$. In [10] the following (Theorem 4) is proved: If, in addition to the standing hypothesis, $f^{-1}(y)$ is locally connected for each $y \in Y$ and $U$ is a nonempty open subset of $X$, then there exists a nonempty open set $V$, such that $\bar{V} \subset U$ and $V$ has property $\mathrm{P}$. This together with the two lemmas that follow will be used in the proof of Theorem 3.

Lemma A. If, in addition to the hypothesis of Theorem 3, $U$ and $V$ are open sets having property $\mathrm{P}$ and $\bar{V} \subset U$, then $U^{\prime}=\left[U-f^{-1} f(\bar{V})\right]+V$ has property $\mathrm{P}$.

Proof. Let $W=U-f^{-1} f(\bar{V})$. Then $U^{\prime}=W+V$ and $f(\bar{W}) \cdot f(V)$ $=f(W) \cdot f(\bar{V})=0$. To show that $U^{\prime}$ satisfies the first part of property P, suppose, (1) $y \in[f(\bar{W})-f(\bar{V})]$. Then $f^{-1}(y) \cdot \bar{U}^{\prime}=f^{-1}(y) \cdot(\bar{W}+\bar{V})$ $=f^{-1}(y) \cdot \bar{W}=f^{-1}(y) \cdot \bar{U}$ which is connected because $U$ has property $\mathrm{P}$. If (2) $y \in f(\bar{W}) \cdot f(\bar{V})$, then $f^{-1}(y) \cdot \bar{U}=f^{-1}(y) \cdot \bar{W}$, so that, by part (1), $f^{-1}(y) \cdot \bar{U}^{\prime}$ is the limit supremum of a sequence $\left\{f^{-1}\left(y_{i}\right) \cdot \bar{U}\right\}$ of con- 
nected sets, where for each positive integer $i, y_{i} \in[f(\bar{W})-f(\bar{V})]$. But each component of this limit supremum contains the nonempty set $f^{-1}(y) \cdot \bar{V}$ and hence it is connected. Finally, (3) if $y \in[f(\bar{V})-f(\bar{W})]$. then $f^{-1}(y) \cdot \bar{U}^{\prime}=f^{-1}(y) \cdot \bar{V}$ which is connected as $V$ has property $\mathrm{P}$,

To show that $U^{\prime}$ satisfies the second part of property $\mathrm{P}$, suppose first that $y \in f(\bar{V})$. Then there exists a point $x$ in $f^{-1}(y) \cdot \bar{V}$ and a neighborhood $N$ of $x$ such that $f^{-1} f(V) \cdot N \subset V$. But $x$ is in the open set $N^{\prime}=U \cdot N$ because $x \in \bar{V} \subset U$. Then $f^{-1} f\left(U^{\prime}\right) \cdot N^{\prime}=\left[f^{-1} f(W)\right.$ $\left.+f^{-1} f(V)\right] \cdot N \cdot U \subset\left[U-f^{-1} f(\bar{V})\right]+V=U^{\prime}$. Secondly, if $y \in f\left(U^{\prime}\right)$ $-f(\bar{V})$, then there exists a point $x \in f^{-1}(y) \cdot \bar{U}$ and a neighborhood $N$ of $x$ such that $f^{-1} f(U) \cdot N \subset U$ and such that $f(N) \cdot f(\bar{V})=0$. Then $f^{-1} f\left(U^{\prime}\right) \cdot N=\left[f^{-1} f(U)-f^{-1} f(\bar{V})\right] \cdot N+f^{-1} f(V) \cdot N \subset\left[U-f^{-1} f(\bar{V})\right]+0 \subset U^{\prime}$.

Lemma B. Suppose, in addition to the hypothesis of Theorem 3, that $U$ and $V$ are nonempty open sets having property $\mathrm{P}, f^{-1} f(\bar{V}) \cdot U=V$, and $\epsilon>0$. Then there exist nonempty open sets $U^{\prime}$ and $V^{\prime}$ such that:

(1) $U^{\prime}$ and $V^{\prime}$ have property $\mathrm{P}$;

(2) $f^{-1} f\left(\bar{V}^{\prime}\right) \cdot U^{\prime}=V^{\prime}$;

(3) $f\left(\bar{U}^{\prime}\right)=f(\bar{U})$;

(4) $U^{\prime}, \bar{V}^{\prime} \subset U$;

(5) if $y \in f\left(V^{\prime}\right)$, then $f^{-1}(y) \cdot \bar{U}^{\prime}$ has diameter less than $\epsilon$; and

(6) $\bar{U} \subset N_{\epsilon}\left(V^{\prime}\right)$ and $f(\bar{U}) \subset N_{\epsilon}\left[f\left(V^{\prime}\right)\right]$.

Proof. There exists a positive number $\delta<\epsilon / 3$, such that if $x, x^{\prime} \in X$ and $\rho\left(x, x^{\prime}\right)<3 \delta$, then $\rho\left[f(x), f\left(x^{\prime}\right)\right]<\epsilon$. Let $W=U-V$. Then, by hypothesis, $W=U-f^{-1} f(\bar{V}) \cdot U=U-f^{-1} f(\bar{V})$, so that $W$ is open in $X$ and $f(W) \cdot f(\bar{V})=0$. There exists a finite set $H \subset W$ such that $W \subset N_{\delta}(H)$. Let $A_{1}, A_{2}, A_{3}, \cdots, A_{n}$ denote the points of $H$. As $Y$ is perfect, the open set $f\left[N_{\delta}\left(A_{2}\right)\right] \cdot f(W)$ contains a point $y_{2}^{\prime} \neq f\left(A_{1}\right)$. Therefore there exists a point $A_{2}^{\prime} \in f^{-1}\left(y_{2}^{\prime}\right) \cdot W \cdot N_{\delta}\left(A_{2}\right)$, such that $f\left(A_{1}^{\prime}\right) \neq f\left(A_{2}^{\prime}\right)$. Inductively, there exist points $A_{3}^{\prime}, A_{4}^{\prime}, \cdots, A_{n}^{\prime}$ such that for each integer $i, 1 \leqq i \leqq n, A_{i}^{\prime} \in W \cdot N_{\delta}\left(A_{i}\right)$ and such that $f\left(A_{i}^{\prime}\right) \neq f\left(A_{j}^{\prime}\right)$ unless $i=j$. Let $H^{\prime}=\left\{A_{1}^{\prime}, \cdots, A_{n}^{\prime}\right\}$. Then $N_{28}\left(H^{\prime}\right)$ $\supset N_{\delta}(H) \supset \bar{W}$. Similarly, there exists a finite set $K^{\prime} \subset V$ such that $V \subset N_{2 \delta}\left(K^{\prime}\right)$ and such that no two points of $K^{\prime}$ have the same image under $f$. Thus there exist open sets $R_{1}, R_{2}, \cdots, R_{m}$ of diameter $<\delta$, such that for each integer $i, 1 \leqq i \leqq m, R_{i}$ contains one and only one of the points of $H^{\prime}+K^{\prime}, \bar{R}_{i}$ lies entirely in one of the sets $W, V$, and such that $f\left(\bar{R}_{i}\right) \cdot f\left(\bar{R}_{j}\right)=0$, unless $i=j$.

Then, by Theorem 4 of [10], for each positive integer $i \leqq m$, there exists a nonempty open set $V_{i}$ having property $\mathrm{P}$, such that $\bar{V}_{i} \subset R_{i}$. Then if $1 \leqq i<j \leqq m, f\left(\bar{V}_{i}\right) \cdot f\left(\bar{V}_{j}\right)=0$. Let $V^{\prime}=U_{i-1}^{m} \quad V_{i}$ and $U^{\prime}=[U$ $\left.-f^{-1} f\left(\bar{V}^{\prime}\right)\right]+V^{\prime}$. The six parts of the conclusion will be proven in numerical order. 
First, if $y \in f\left(\bar{V}^{\prime}\right)$, then $f^{-1}(y) \cdot \bar{V}^{\prime}=f^{-1}(y) \cdot \bar{V}_{i}$ for some positive integer $i$, so that $V^{\prime}$ has property $\mathrm{P}$; then by Lemma $\mathrm{A}, U^{\prime}$ has property P. By the definition of $U^{\prime},(2), f^{-1} f\left(\bar{V}^{\prime}\right) \cdot U^{\prime}=V^{\prime}$, and (3), $f\left(\bar{U}^{\prime}\right)$ $=\left[f(\bar{U})-f\left(\bar{V}^{\prime}\right)\right]+f\left(\bar{V}^{\prime}\right)=f(\bar{U})$, and $(4), U^{\prime} \subset U$ and $\bar{V}^{\prime}=\bigcup_{i=1}^{m} \bar{V}_{i} \subset U$. If $y \in f\left(V^{\prime}\right)$, then $f^{-1}(y) \cdot \bar{U}^{\prime}=f^{-1}(y) \cdot \bar{V}^{\prime}=f^{-1}(y) \cdot \bar{V}_{i}$ for some positive integer $i$, so that $(5), f^{-1}(y) \cdot \bar{U}^{\prime}$ has diameter less than $\epsilon$. Lastly, $N_{3 \delta}\left(V^{\prime}\right) \supset N_{2 \delta}\left(H^{\prime}+K^{\prime}\right) \supset \bar{W}+\bar{V} \supset \bar{U}$ so that, by the definition of $\delta$, (6), $N_{\epsilon}\left(V^{\prime}\right) \supset \bar{U}$ and $N_{\epsilon}\left[f\left(V^{\prime}\right)\right] \supset f(\bar{U})$.

TheOREM 3. If, in addition to the standing hypothesis, $Y$ is perfect and for each $y \in Y, f^{-1}(y)$ is connected and locally connected, then there exists a closed subset $X^{\prime}$ of $X$, such that $f \mid X^{\prime}$ is monotone onto $Y$ and such that if $X^{\prime \prime}$ is a proper closed subset of $X^{\prime}$, then $f \mid X^{\prime \prime}$ is not onto $Y$.

PROoF. Let $U_{1}=V_{1}=X$ and for each positive integer $n$ let $\epsilon_{n}=1 / n$ Then $U_{1}$ and $V_{1}$ have property $\mathrm{P}$, and $f^{-1} f\left(\bar{V}_{1}\right) \cdot U_{1}=V_{1}$, so that Lemma B applies. Therefore there exist nonempty open sets $U_{2}, V_{2}$ satisfying the six conditions of Lemma $\mathrm{B}$ relative to $U_{1}, V_{1}$, and $\epsilon_{1}$. But parts (1) and (2) of Lemma B allow it to be applied to $U_{2}, V_{2}$, and $\epsilon_{2}$, and so on indefinitely. Therefore there exist two sequences $\left\{U_{i}\right\}_{i=1}^{\infty}$ and $\left\{V_{i}\right\}_{i=1}^{\infty}$ of open sets such that for each positive integer $i$ :

(1) $U_{i}$ and $V_{i}$ have property $\mathrm{P}$;

(2) $f^{-1} f\left(\bar{V}_{i}\right) \cdot U_{i}=V_{i}$;

(3) $f\left(\bar{U}_{i}\right)=f\left(\bar{U}_{i-1}\right)=\cdots=f\left(\bar{U}_{1}\right)=Y$;

(4) $U_{i+1}, \bar{V}_{i+1} \subset U_{i}$;

(5) if $y \in f\left(V_{i+1}\right)$, then $f^{-1}(y) \cdot \bar{U}_{i+1}$ has diameter less than $\epsilon_{i+1}$; and

(6) $\bar{U}_{i} \subset N_{\epsilon_{i+1}}\left[\left(V_{i+1}\right)\right]$ and $f\left(\bar{U}_{i}\right)=Y \subset N_{\epsilon_{i+1}}\left[f\left(V_{i+1}\right)\right]$.

Let $C=\left\{x \mid\right.$ for infinitely many integers $\left.i, x \in V_{i}\right\}$. From part (6) it follows that if $N$ is a nonempty open set in $Y$ and $n>0$, then there exists an integer $i>n$ and a nonempty open set $R$ such that $\bar{R} \subset f\left(V_{i}\right)$ and $\bar{R} \subset N$. By repeated application of this fact, (2), and (4), it follows that $f(C)$ intersects each open subset of $Y$ and hence $f(\bar{C})=Y$. Furthermore, for each positive integer $n, V_{n} \subset N_{\epsilon_{n}}(\bar{C})$. For suppose $x \in V_{n}$ and let $y=f(x)$. As $y \in f(\bar{C})$, then $f^{-1}(y)$ contains a point $x^{\prime}$ of $\bar{C}$. But $x^{\prime}$ must be in $\widetilde{U}_{n}$ because for each integer $i>n, V_{i} \subset U_{n}$ and hence $C \subset U_{n}$. Then $x, x^{\prime} \in f^{-1}(y) \cdot \bar{U}_{n}$, which, by (5), has diameter less than $\epsilon_{n}$.

Let $X^{\prime}=\bigcap_{i=1}^{\infty} \bar{U}_{i}$; then $X^{\prime}=\bar{C}$. Because, firstly, $C \subset U_{i}$ for each positive integer $i$ and hence $\bar{C} \subset X^{\prime}$. Secondly, since $V_{i+1} \subset N_{\epsilon_{i+1}}(\bar{C})$ for each positive integer $i$, and since by (6), $\bar{U}_{i} \subset N_{\epsilon_{i+1}}\left(V_{i+1}\right)$, it follows that $\bar{U}_{i} \subset N_{2 \epsilon_{i+1}}(\bar{C})$ for each positive integer $i$. Hence $X^{\prime} \subset \bar{C}$.

That $f \mid X^{\prime}$ is monotone is a consequence of the fact that for each positive integer $i, U_{i}$ has property $\mathrm{P}$ and hence $f \mid \bar{U}_{i}$ is monotone. By part (3), $f\left(X^{\prime}\right)=Y$. Lastly, if $X^{\prime \prime}$ is a proper closed subset of $X$, then 
$X^{\prime \prime}$ does not contain $C$. Therefore it will complete the proof to show that if $x \in C$, then $f \mid X^{\prime}$ is one-to-one at $x$. But for infinitely many integers $i, f(x) \in f\left(V_{i}\right)$ and hence $f^{-1} f(x) \cdot \bar{U}_{i}$ has diameter less than $\epsilon_{i}$. Therefore $f^{-1} f(x) \cdot X^{\prime}$ contains only one point.

CoROllary. If, in addition to the hypothesis of Theorem 3, $Y$ is a continuum irreducible between two of its points, $A$ and $B$, then there exists a sub-continuum $X^{\prime}$ of $X$, such that $f \mid X^{\prime}$ is monotone, onto $Y$, and such that $X^{\prime}$ is irreducible between $f^{-1}(A) \cdot X^{\prime}$ and $f^{-1}(B) \cdot X^{\prime}$.

Proof. By Theorem 3 there exists a closed subset $X^{\prime}$ of $X$ such that $f \mid X^{\prime}$ is monotone onto $Y$, and such that no proper closed subset of $X^{\prime}$ maps onto $Y$ under $f$. As $Y$ is a continuum, so also is $X^{\prime}$. As the image of a subcontinuum of $X^{\prime}$ intersecting $f^{-1}(A)$ and $f^{-1}(B)$ would contain $A$ and $B$ and hence have to contain all of $Y$, then $X^{\prime}$ must be irreducible between $A$ and $B$.

\section{BIBLIOGRAPHY}

1. R. D. Anderson, On monotone interior mappings in the plane, Trans. Amer. Math. Soc. vol. 73 (1952) p. 211.

2. D. van Dantzig, Über topologisch homogene Kontinua, Fund. Math. vol. 15 (1930) p. 102.

3. Eldon Dyer, Irreducibility of the sum of the elements of a continuous collection of continua, Duke Math. J. vol. 20 (1953) p. 589.

4. M. E. Hamstrom, Concerning continuous collections of continuous curves, Proc. Amer. Math. Soc. vol. 4 (1953) p. 240.

5. B. Knaster, Un continu irréductible d décomposition continue en tranches, Fund. Math. vol. 25 (1935) p. 577.

6. E. E. Moise, $A$ theorem on monotone interior transformations, Bull. Amer. Math. Soc. vol. 25 (1949) p. 810.

7. J. Rozanska, Über stetige Abbildungen eines Elementes, Fund. Math. vol. 28 (1937) p. 266.

8. G. T. Whyburn, Arc-preserving transformations, Amer. J. Math. vol. 58 (1936) p. 305 .

9. - On irreducibility of transformations, Amer. J. Math. vol. 61 (1939) p. 820.

10. R. F. Williams, Local properties of open mappings, Duke Math. J. vol. 22 (1955) p. 339.

11. W. A. Wilson, On the structure of a continuum, limited and irreducible between two points, Amer. J. Math. vol. 48 (1926) p. 147.

UNIVERSITY OF VIRGINIA AND

Florida State University 